\title{
Recent progresses on the new condensed forms of single-walled carbon nanotubes and energy-harvesting devices
}

\author{
CHEN MinJiang ${ }^{1,2}$, YU Fang $^{1}$, HU LiJun $^{1} \&$ SUN LianFeng ${ }^{1 *}$ \\ ${ }^{1}$ National Center for Nanoscience and Technology, Beijing 100190, China; \\ ${ }^{2}$ Graduate School of Chinese Academy of Sciences, Beijing 100049, China
}

Received May 10, 2011; accepted July 20, 2011; published online October 28, 2011

\begin{abstract}
We demonstrate an effective method to prepare a new condensed form of single-walled carbon nanotubes (crystal of SWNTs) using a series of diamond wire drawing dies. X-ray diffraction indicates that the SWNTs form a two-dimensional triangular lattice with a lattice constant of $19.62 \AA$. An intertube spacing of $3.39 \AA$ of between adjacent SWNTs results in a sharp (002) reflection in the X-ray diffraction pattern. Meanwhile, we developed an approach based on the Coulomb explosion to separate SWNTs from their bundle. The separated SWNTs have a typical length of several microns and form a nanotree at one end of the original bundle. The separation is convenient and involves no surfactant. In studying devices comprising SWNTs, we find that a four-probe technique can be employed to study the filling of and flow within the inner channel of an individual SWNT. Current/voltage can drive water molecules to have directional flow along an SWNT, and the flowing of water inside an SWNT can induce a voltage gradient force (an induced electromotive force) along the SWNT. This energy conversion is realized by the mutual coupling of water dipoles and charge carriers present in SWNTs. The results suggest that SWNTs can be exploited as molecular channels for water and may find potential application in nanoscale energy conversion. Moreover, a surface-energy generator comprising SWNTs was demonstrated to harvest the surface energy of ethanol. The performance (the induction rate for $V_{\mathrm{oc}}$, the value of $V_{\mathrm{oc}}$ and the output power) can be significantly enhanced by the Marangoni effect.
\end{abstract}

single-walled carbon nanotube (SWNT), crystal of SWNT, SWNT tree, energy conversion, surface-energy generator

Citation: Chen M J, Yu F, Hu L J, et al. Recent progresses on the new condensed forms of single-walled carbon nanotubes and energy-harvesting devices. Chin Sci Bull, 2012, 57: 181-186, doi: 10.1007/s11434-011-4818-z

Since its discovery by Iijima in 1991, the carbon nanotube has attracted much attention because of its remarkable phenomena related to the nanoscale confinement, which lead to the possibility of developing a wide range of applications of new materials and advanced devices. Many interesting phenomena of single-walled carbon nanotubes (SWNTs), which have smaller diameters than multiwalled nanotubes, have been demonstrated; e.g., the Aharonov-Bohm effect [1], Coulomb blocking effect [2-4], and Josephson effect $[5,6]$. It is well known that fullerenes have their most interesting properties when in their condensed form [7,8]; e.g., superconductivity in alkali-doped $\mathrm{C}_{60}$. Theoretical works predict that this may also be true for carbon nanotubes

*Corresponding author (email: slf@nanoctr.cn)
$[9,10]$. However, in experiments, it is still a great challenge to grow SWNTs with identical diameters and chirality; hence, it is not possible to obtain a crystalline form of SWNTs. A highly densely packed SWNT array (SWNT solid) [11] has been achieved by exploiting the liquidinduced collapse of SWNT forests [12], while high-performance fibers [13] have been obtained by the direct spinning of carbon nanotube fibers [14]. For these two materials, although a peak of (002) was observed in the X-ray diffraction (XRD) pattern, there is diffuse intensity at angles below the (002) peak. This indicates that some of the interlayer spacings are greater than those typical of graphene stacks. Recently, we found that SWNTs form a crystalline structure via the van der Waals force when they are perfectly aligned and ideally densely packed after treating with a series of 
diamond wire drawing dies [15]. This work opens a new research field of the condensed form of SWNTs and possible superconductivity in crystals of SWNT after proper doping.

In this review, we also present another interesting form of SWNTs: a nanotree of SWNTs obtained by Coulomb explosion of their bundle [16]. Furthermore, we also introduce energy-related devices of individual SWNTs and a rope of SWNTs: individual water-filled SWNTs as hydroelectric power converters [17] and a surface-energy generator comprising SWNTs [18], respectively.

\section{New condensed form of SWNTs}

\subsection{Crystal of SWNTs}

SWNTs are usually grown using one of three techniques: arc discharge [19], laser ablation [20], or chemical vapor deposition [21]. The SWNTs grown using these methods are a mixture of individual SWNTs with different diameters and chirality. Meanwhile, the SWNTs are usually entangled and poorly ordered. We established an inexpensive and effective method to align SWNTs using a series of diamond wire drawing dies [15]. As shown in Figure 1, during each drawing, the SWNTs form a bundle with the same diameter as that of the die used. The SWNTs produced are gray with a metallic luster.

From scanning electron microscopy (SEM) images of the SWNT bundles, it is seen that the SWNTs fabricated in this way are perfectly aligned and highly densely packed (HDPA-SWNTs) as shown in Figure 2(a). High-resolution transmission electron microscopy (HRTEM) also reveals the as-fabricated SWNTs have a perfectly aligned microstructure as shown in Figure 2(b). It should be noted that these bundles are composed of dense SWNTs, and the number of SWNTs in each bundle is estimated to be as high as $10^{6}$. These SWNT materials are clean and free of amorphous carbon.

The XRD pattern of these HDPA-SWNTs differs from that of untreated SWNTs, as shown in Figure 3(a). At a low angle in the XRD pattern, a strong and discrete peak appears at $2 \theta=5.20^{\circ}$. This Bragg peak can be indexed to (10) diffraction $\left(d_{(10)}\right.$-spacing of $\left.16.97 \AA\right)$ for a two-dimensional triangular lattice of SWNTs. Therefore, the lattice constant, $a=19.62 \AA$, is obtained by the equation $a \times \sqrt{3} / 2=d_{(10)}$-spacing. The following four relatively broad peaks can be indexed as (11), (20), (30) and (40). Another interesting and important feature of the XRD pattern is that there is a very sharp and strong peak at $2 \theta=$ $26.3^{\circ}$ corresponding to a $d$-spacing of $3.39 \AA$ and lattice constant $a=19.62 \AA$, which is believed to arise from the nearly ideal graphitic packing of SWNTs due to van der Waals interaction. The sharp (002) reflection arises from an intertube spacing of $3.39 \AA$ for adjacent SWNTs, and it is not observed for individual SWNTs or small crystalline ropes of SWNTs. Therefore, these highly dense and perfectly aligned SWNTs (HDPA-SWNTs) are a condensed and crystalline form of SWNTs.

The crystal of SWNTs and the as-grown SWNTs film were characterized by micro-Raman spectroscopy with 632 $\mathrm{nm}$ laser excitation, as shown in Figure 3(b). In comparison with the as-grown SWNTs, the radial breathing mode of the SWNT crystal changes significantly: the radial breathing mode signals for larger-diameter SWNTs HDPA-SWNTs are suppressed. This suppression may result from the stronger coupling between the neighboring SWNTs in the crystal of SWNTs, which results from smaller intertube spacing of $3.39 \AA$ compared with $9 \AA$ in the SWNT solid [17].

\subsection{Nanotree of SWNTs}

SWNTs are often entangled with each other and readily form bundles with a large van der Waals binding energy $(\sim 500 \mathrm{eV} / \mu \mathrm{m}$ of tube-tube contact) $[22,23]$. This has been a major obstacle for potential applications of SWNTs in nanodevices and nanoelectronic circuits. To overcome this limitation, many researchers have explored selective chemistry to obtain monodispersed SWNTs [24-27]. These chemical approaches have doping effects on SWNTs $[28,29]$, and the obtained individual SWNTs are usually very short. We developed a novel effect of Coulomb charging to separate nanotubes from their bundles, and this simple physical technique should be performed directly on the substrates to avoid chemical modification [16].

Figure 4 is a typical SEM image of an SWNT rope, SWNT bundles, and an SWNT nanotree. The SWNTs mainly separated at one end of an SWNT bundle, and radiated
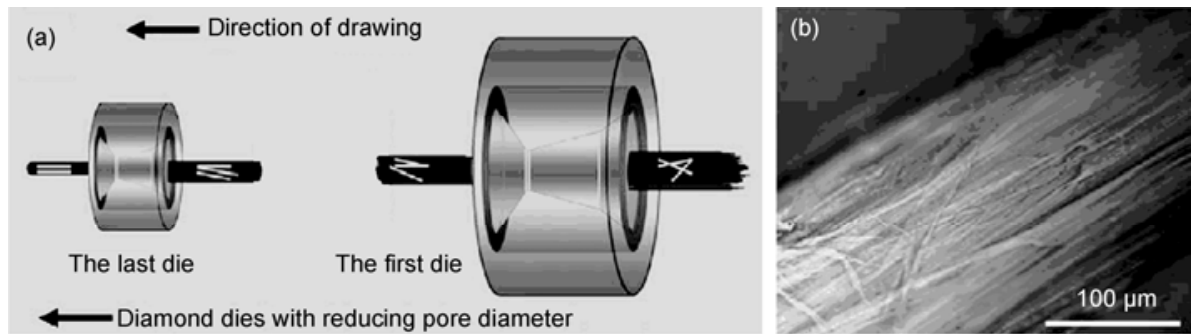

Figure 1 Alignment of SWNTs. (a) Schematic diagram of the diamond wire drawing dies used to align SWNTs. (b) Optical image of SWNTs after treating. 


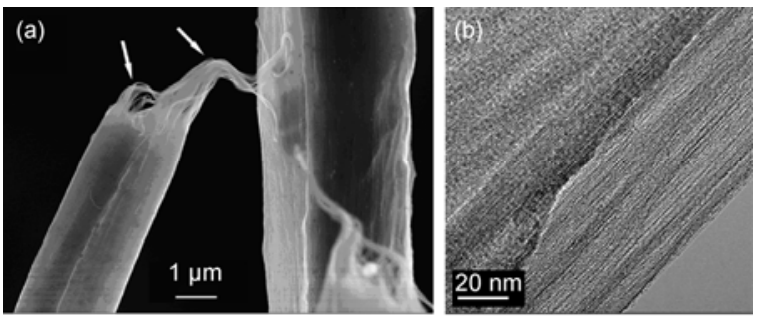

Figure 2 (a) SEM image of two bundles of SWNTs. The good flexibility of SWNTs is shown by the white arrows. (b) HRTEM image of a crystal of SWNTs.
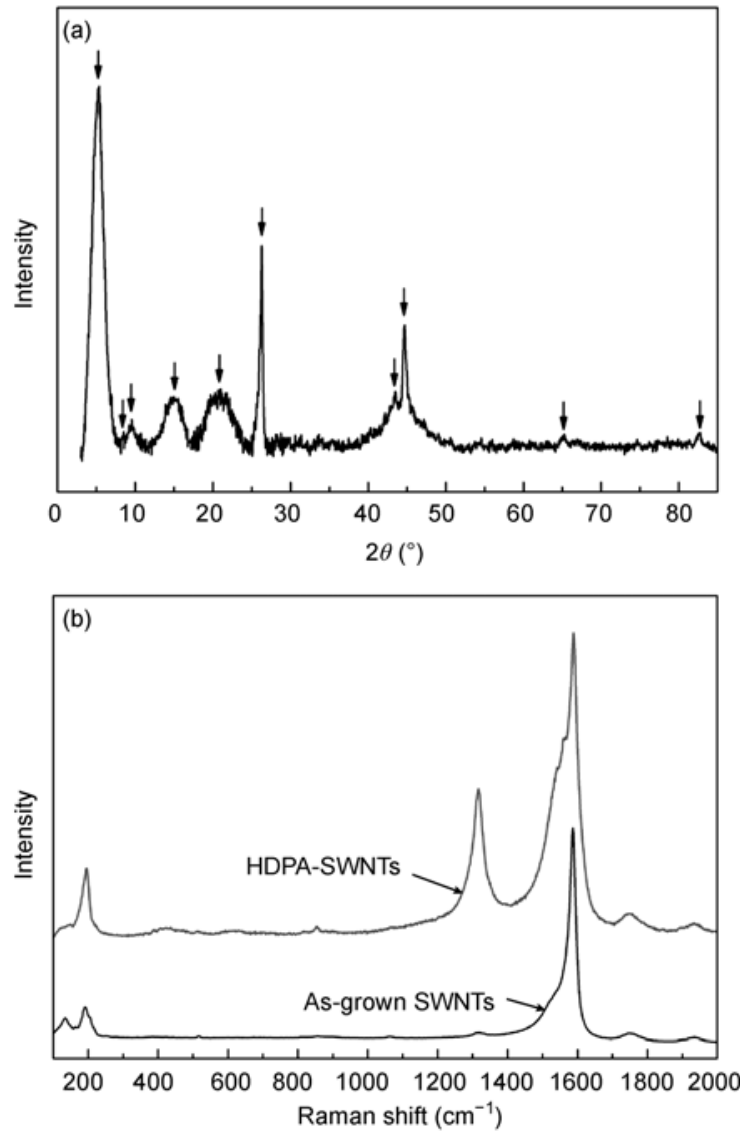

Figure 3 (a) XRD patterns of HDPA-SWNTs after subtraction of the background. (b) Raman spectra of the as-grown SWNTs and the HDPA-SWNTs.

from one center. These isolated SWNTs are relatively straight and separated from each other as far as possible. The mechanism of the formation of these nanotrees is as follows. Before the individual SWNTs are charged, they are linked to each other with van der Waals binding energy. When the SWNTs are positively charged, a Coulomb repulsion and van der Waals force exist simultaneously. As the electrostatic potential increases, the increasing charge induces Coulomb explosion and the separation of SWNTs.

Interestingly, we also observed similar morphologies of SWNTs at breaking points resulting from electrical heating in a vacuum, as shown in Figure 5. After heating with a DC

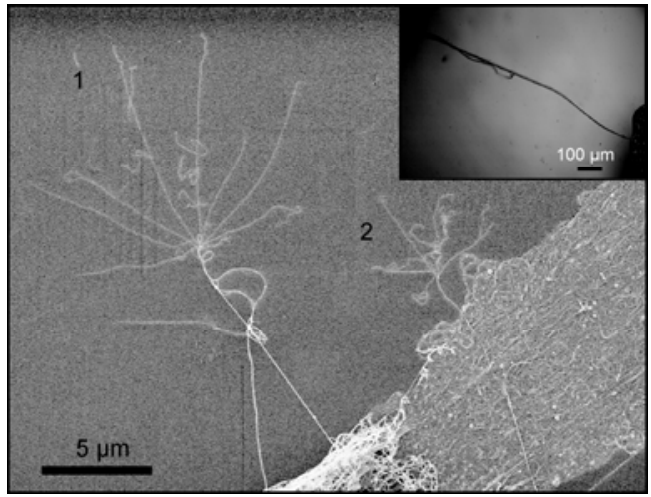

Figure 4 SEM image of two typical nanotrees of SWNTs formed at the ends of two SWNT bundles via Coulomb explosion. The inset is an optical image.
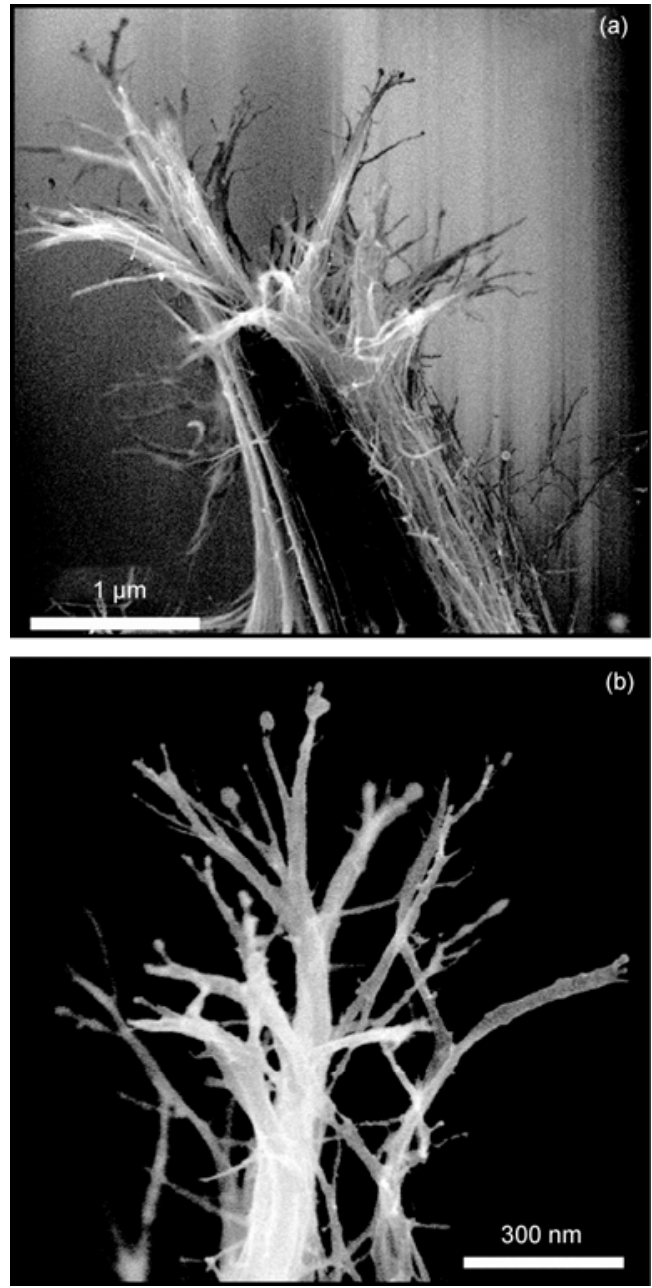

Figure 5 SEM images of typical nanotrees of SWNTs formed at the breaking points of an SWNT bundle.

bias for about $10 \mathrm{~min}$, the SWNT bundle radiated light and finally broke down because of Joule heating. At the tips of the SWNTs tree, there are spheres that are believed to be due to the surface tension produced by the melted carbon and/or catalyst at the breakdown instant [30]. 


\section{Energy transformation devices comprising SWNTs}

\subsection{Individual water-filled SWNTs as hydroelectric power converters}

Water flowing inside/outside SWNTs is a unique nanofluidic system in which the water molecules and the walls of the SWNTs form an intimate interface at the atomic level. It is possible that the coupling between water dipoles and free charge carriers in the nanotubes is mutual [31-34], and a constant current can induce a directional water flow inside the nanotube. We find that a significant voltage difference can be detected at one part of an individual SWNT device when a current is applied at another part of the same SWNT after the device is exposed to water vapor [17], as shown in Figure 6.

The current-voltage characteristics of the device were measured, as shown in Figure 7. In a vacuum, $V_{1}$ and $V_{2}$ are nonzero and equal to each other. When the device is exposed to water vapor, $V_{1}$ and $V_{2}$ are no longer equal to each other, and a voltage difference $\left(\delta V=V_{1}-V_{2}\right)$ is thus measured in the "generator" part of the device. $\delta V$ has a linear dependence on the current applied to the "motor" part.

\subsection{Surface-energy generator comprising SWNTs}

Surface energy plays an important role in surface physics, biophysics, surface chemistry, and catalysis [35,36]. A gradient of surface energy between a solid and liquid interface can induce the transport of liquids [37-39]. For low power of nanomaterials, it is attractive to use the surface energy as an energy source at the nanoscale. We demonstrate an effective design of SWNTs to harvest surface energy of ethanol and convert it into electricity. A rope of SWNTs can be used to study the coupling between liquid outside SWNTs with carriers of SWNTs [18].

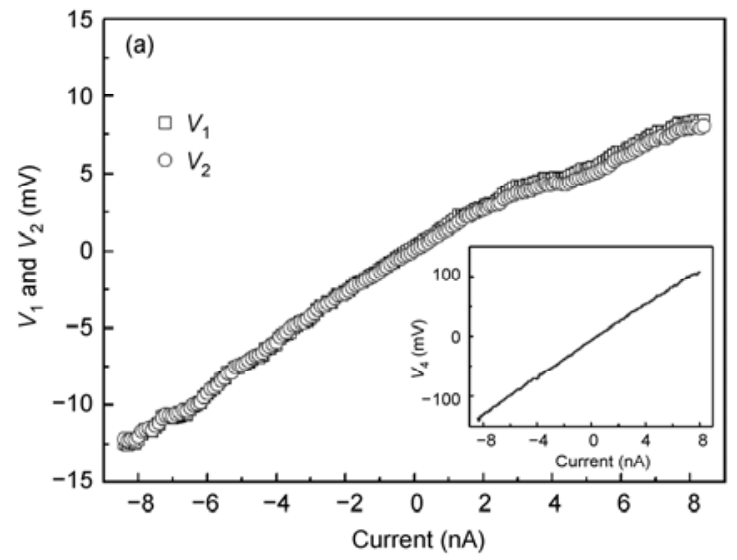
consumption of nanodevices and high specific surface area

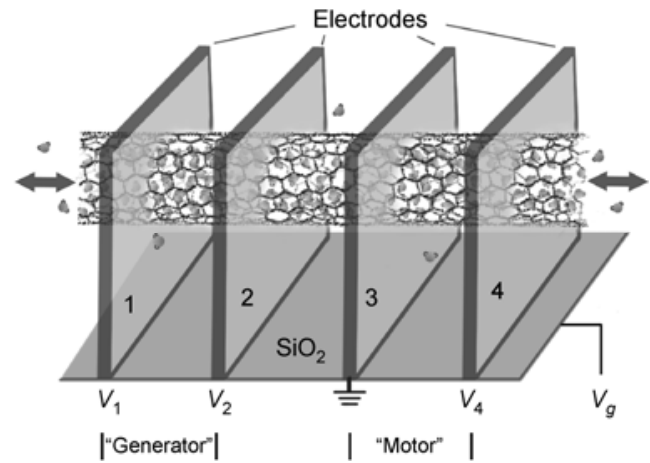

Figure 6 Schematic diagram of the hydroelectric power converter. Current/voltage is applied between electrodes 3 and 4 (the "motor" part) and the induced voltage difference is measured between electrodes 1 and 2 (the "generator" part) when the device is exposed to water vapor.

In an open beaker, $V_{\mathrm{oc}}$ is initially zero (Figure 8(a)). When the ethanol level reaches the SWNT rope, $V_{\text {oc }}$ begins to increase linearly from zero to $200 \mu \mathrm{V}$ for the first $240 \mathrm{~s}$, and then saturates at $219 \mu \mathrm{V}$, where it remains constant for over $6 \mathrm{~h}$. The mechanism relating to these observation is that ethanol molecules are pulled up along the channels formed among individual SWNTs by the capillary force; thus, $V_{\mathrm{oc}}$ is induced by the coupling between the charge carriers of the SWNTs and the ethanol molecules flowing at the interface $[33,40]$. When the beaker is covered, $V_{\text {oc }}$ gradually decreases to its original value (Figure 8(b)). This process can be repeated and the characteristics of varying $V_{\text {oc }}$ can be well reproduced.

If an SWNT rope is pretreated with deionized water, a very sharp increase in $V_{\mathrm{oc}}$ is observed when the ethanol level reaches the SWNT rope, as shown in Figure 9. $V_{\text {oc }}$ jumps to $853 \mu \mathrm{V}$ in less than $1 \mathrm{~s}$. $V_{\text {oc }}$ changes much more quickly and by a much larger magnitude compared with $V_{\text {oc }}$ of an untreated device. Ethanol has lower surface tension than water, and the difference provides an additional driving force for ethanol to climb up along the channels among the SWNTs, which is called the Marangoni effect [41-43].

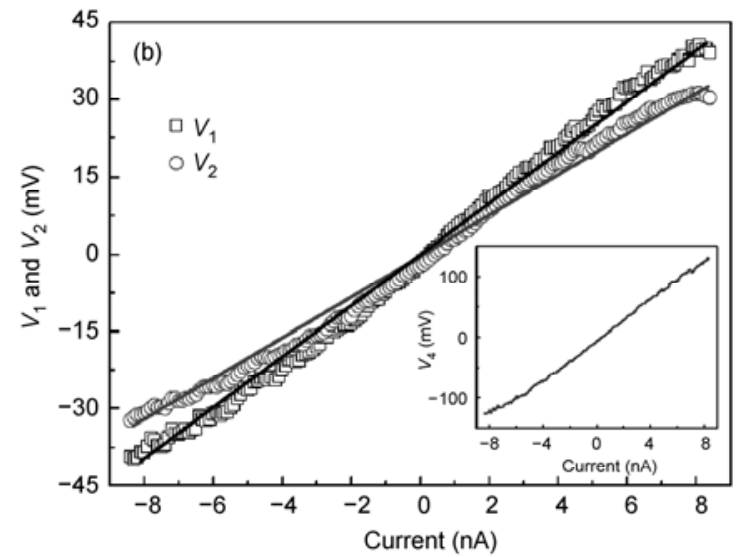

Figure 7 (a) In a vacuum, $V_{1}$ and $V_{2}$ are almost equal and have linear dependence on the current applied. (b) If water vapor is present in the chamber, there is a significant difference between $V_{1}$ and $V_{2}$. 

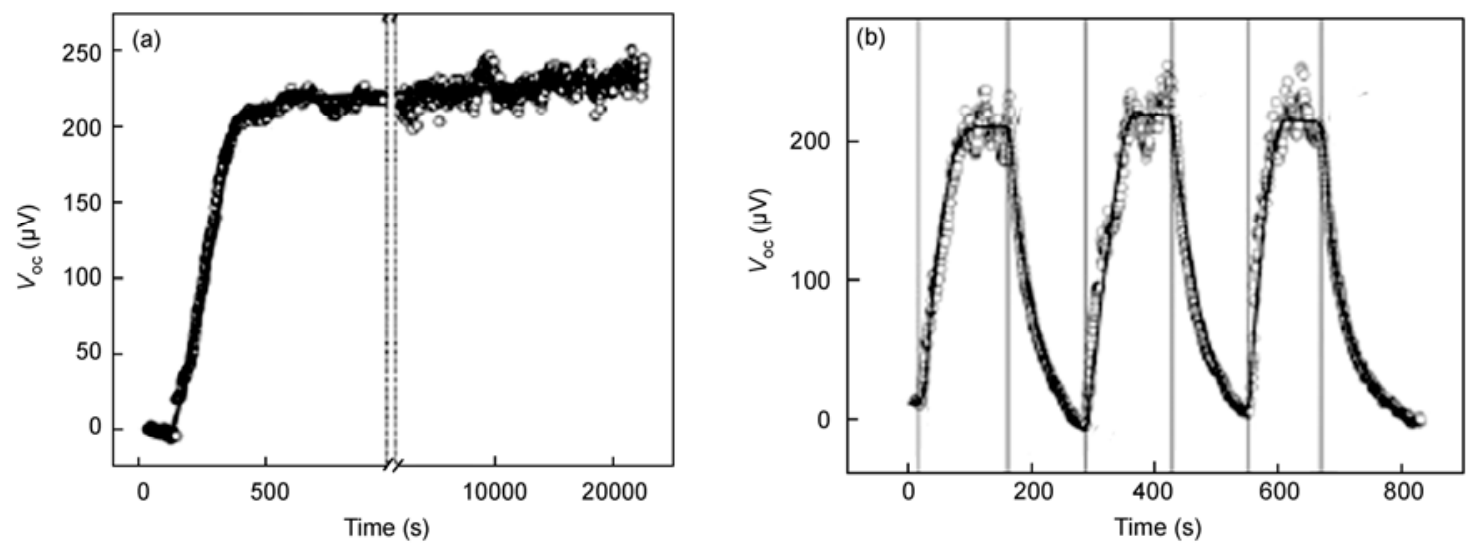

Figure 8 Dynamic characteristics of $V_{\text {oc }}$. (a) Zero $V_{\text {oc }}$ is observed until the ethanol level reaches the SWNT rope. $V_{\text {oc }}$ increases to a saturated value of 219 $\mu \mathrm{V}$ in about $300 \mathrm{~s}$. (b) $V_{\mathrm{oc}}$ decreases gradually back to its original value when the beaker is covered as indicated by region 2 .

(a)

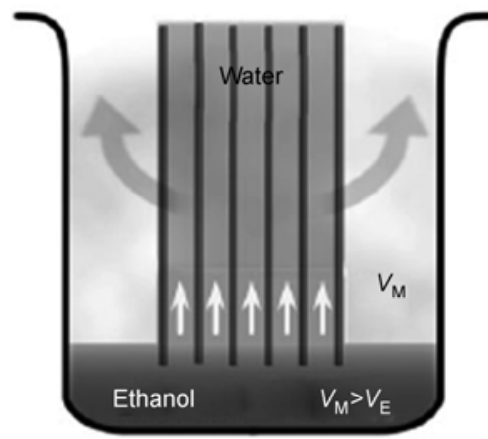

(b)

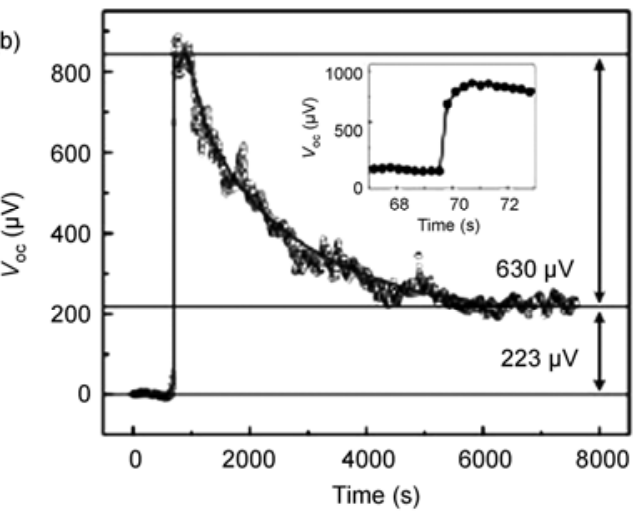

Figure 9 (a) If the SWNT is pretreated with water, the flowing velocity of ethanol is increased by the Marangoni effect. (b) Once the SWNT is pretreated with water, $V_{\text {oc }}$ jumps to $653 \mu \mathrm{V}$ in less than $1 \mathrm{~s}$, indicating much faster induction and a larger value of $V_{\text {oc }}$ for the surface energy generator owing to the Marangoni effect.

\section{Conclusion}

In summary, an inexpensive and effective method to prepare a crystal of SWNTs is demonstrated using a series of diamond wire drawing dies. XRD indicates that the SWNTs form a two-dimensional triangular lattice. Additionally, a sharp (002) reflection is seen in the XRD pattern. Meanwhile, we developed an approach based on the Coulomb explosion to separate SWNTs from their bundle. This technique is convenient and involves no surfactant. In studies of devices comprising SWNTs, we find that current/voltage can drive water molecules to have directional flow along SWNTs, and the flowing of water inside an SWNT can induce a voltage gradient force (an induced electromotive force) the SWNT. The results suggest that SWNTs can be exploited as molecular channels for water and may find potential application in nanoscale energy conversion. Moreover, a surface-energy generator (SEG) comprising SWNTs was demonstrated to harvest the surface energy of ethanol. The performance (the induction rate for $V_{\text {oc }}$, the value of $V_{\mathrm{oc}}$ and the output power) can be significantly enhanced by the Marangoni effect.
This work was supported by the National Natural Science Foundation of China (10774032, 90921001 and 50952009).

1 Ajiki H, Ando T. Electronics states of carbon nanotubes. J Phys Soc Jpn, 1993, 62: 1255-1266

2 Tans S J, Devoret M H, Dai H J, et al. Individual single-wall carbon nanotubes as quantum wires. Nature, 1997, 386: 474-477

3 Tans S J, Devoret M H, Groeneveld R J A, et al. Electron-electron correlations in carbon nanotubes. Nature, 1998, 394: 761-764

4 Bezryadin A, Verschueren A R M, Tans S J, et al. Multiprobe transport experiments on individual single-wall carbon nanotubes. Phys Rev Lett, 1998, 80: 4036-4039

5 Kasumov A Y, Deblock R, Kociak M, et al. Supercurrents through single-walled carbon nanotubes. Science, 1999, 284: 1508-1511

6 Fazio R, Hekking F W J, Odintsov A A. Josephson current through a luttinger liquid. Phys Rev Lett, 1995, 74: 1843-1846

7 Kratschmer W, Lamb L D, Fostiropoulos K, et al. Solid C60: A new form of carbon. Nature, 1990, 347: 354-358

8 Hebard A F, Rosseinsky M J, Haddon R C, et al. Superconductivity at 18-K in potassium-doped C60. Nature, 1991, 350: 600-601

9 Delaney P, Choi H J, Ihm J, et al. Broken symmetry and pseudogaps in ropes of carbon nanotubes. Nature, 1998, 391: 466-468

10 Tersoff J, Ruoff R S. Structural-properties of a carbon-nanotube crystal. Phys Rev Lett, 1994, 73: 676-679

11 Futaba D N, Hata K, Yamada T, et al. Shape-engineerable and highly densely packed single-walled carbon nanotubes and their application as super-capacitor electrodes. Nat Mater, 2006, 5: 987-994 
12 Hata K, Futaba D N, Mizuno K, et al. Water-assisted highly efficient synthesis of impurity-free single-walled carbon nanotubes. Science, 2004, 306: 1362-1364

13 Motta M, Moisala A, Kinloch I A, et al. High performance fibres from 'Dog bone' carbon nanotubes. Adv Mater, 2007, 19: 3721-3726

14 Li Y L, Kinloch I A, Windle A H. Direct spinning of carbon nanotube fibers from chemical vapor deposition synthesis. Science, 2004, 304: 276-278

15 Liu G T, Zhao Y C, Deng K, et al. Highly dense and perfectly aligned single-walled carbon nanotubes fabricated by diamond wire drawing dies. Nano Lett, 2008, 8: 1071-1075

16 Liu G T, Zhao Y C, Zheng K H, et al. Coulomb explosion: A novel approach to separate single-walled carbon nanotubes from their bundle. Nano Lett, 2009, 9: 239-244

17 Zhao Y C, Song L, Deng K, et al. Individual water-filled singlewalled carbon nanotubes as hydroelectric power converters. Adv Mater, 2008, 20: 1772-1776

18 Liu Z, Zheng K H, Hu L J, et al. Surface-energy generator of singlewalled carbon nanotubes and usage in a self-powered system. Adv Mater, 2010, 22: 999-1003

19 Journet C, Maser W K, Bernier P, et al. Large-scale production of single-walled carbon nanotubes by the electric-arc technique. Nature, 1997, 388: 756-758

20 Thess A, Lee R, Nikolaev P, et al. Crystalline ropes of metallic carbon nanotubes. Science, 1996, 273: 483-487

21 Cheng H M, Li F, Su G, et al. Large-scale and low-cost synthesis of single-walled carbon nanotubes by the catalytic pyrolysis of hydrocarbons. Appl Phys Lett, 1998, 72: 3282-3284

22 Girifalco L A, Hodak M, Lee R S. Carbon nanotubes, buckyballs, ropes, and a universal graphitic potential. Phys Rev B, 2000, 62: 13104-13110

23 O'Connell M J, Bachilo S M, Huffman C B, et al. Band gap fluorescence from individual single-walled carbon nanotubes. Science, 2002, 297: 593-596

24 Doorn S K, Fields R E, Hu H, et al. High resolution capillary electrophoresis of carbon nanotubes. J Am Chem Soc, 2002, 124: 3169-3174

25 Heller D A, Mayrhofer R M, Baik S, et al. Concomitant length and diameter separation of single-walled carbon nanotubes. J Am Chem Soc, 2004, 126: 14567-14573

26 Chattopadhyay D, Lastella S, Kim S, et al. Length separation of Zwitterion-functionalized single wall carbon nanotubes by GPC. J Am Chem Soc, 2002, 124: 728-729
27 Niyogi S, Hu H, Hamon M A, et al. Chromatographic purification of soluble single-walled carbon nanotubes (s-SWNTs). J Am Chem Soc, 2001, 123: 733-734

28 Kamaras $\mathrm{K}$, Itkis $\mathrm{M} \mathrm{E}, \mathrm{Hu} \mathrm{H}$, et al. Covalent bond formation to a carbon nanotube metal. Science, 2003, 301: 1501

29 Shin H J, Kim S M, Yoon S M, et al. Tailoring electronic structures of carbon nanotubes by solvent with electron-donating and -withdrawing groups. J Am Chem Soc, 2008, 130: 2062-2066

30 Wei Y, Jiang K L, Liu L, et al. Vacuum-breakdown-induced needleshaped ends of multiwalled carbon nanotube yarns and their field emission applications. Nano Lett, 2007, 7: 3792-3797

31 Maiti A, Andzelm J, Tanpipat N, et al. Effect of adsorbates on field emission from carbon nanotubes. Phys Rev Lett, 2001, 87: 155502155505

32 Lu D Y, Li Y, Rotkin S V, et al. Finite-size effect and wall polarization in a carbon nanotube channel. Nano Lett, 2004, 4: 23832387

33 Ghosh S, Sood A K, Kumar N. Carbon nanotube flow sensors. Science, 2003, 299: 1042-1044

34 Ghosh S, Sood A K, Ramaswamy S, et al. Flow-induced voltage and current generation in carbon nanotubes. Phys Rev B, 2004, 70: 205423-205427

35 Hu D L, Bush J W M. Meniscus-climbing insects. Nature, 2005, 437: 733-736

$36 \mathrm{Hu}$ D L, Chan B, Bush J W M. The hydrodynamics of water strider locomotion. Nature, 2003, 424: 663-666

37 Grunze M. Surface science-Driven liquids. Science, 1999, 283: 41-42

38 Gallardo B S, Gupta V K, Eagerton F D, et al. ELectrochemical principles for active control of liquids on submillimeter scales. Science, 1999, 283: 57-60

39 Ichimura K, Oh S K, Nakagawa M. Light-driven motion of liquids on a photoresponsive surface. Science, 2000, 288: 1624-1626

40 Kral P, Shapiro M. Nanotube electron drag in flowing liquids. Phys Rev Lett, 2001, 86: 131-134

41 Scriven L E, Sternling C V. The marangoni effects. Nature, 1960, 187: $186-188$

42 Cai Y, Newby B M Z. Marangoni flow-induced self-assembly of hexagonal and stripelike nanoparticle patterns. J Am Chem Soc, 2008 , 130: 6076-6077

43 Cazabat A M, Heslot F, Troian S M, et al. Fingering instability of thin spreading films driven by temperature gradients. Nature, 1990, 346: $824-826$

Open Access This article is distributed under the terms of the Creative Commons Attribution License which permits any use, distribution, and reproduction in any medium, provided the original author(s) and source are credited. 